\title{
Rationality of certain cuspidal unipotent representations in crystalline cohomology groups
}

\author{
Joujuu OHMORI \\ (Received October 5, 2004; Revised May 24, 2005)
}

\begin{abstract}
We complete the determination of the local Schur indices of each unipotent representation of the group $G\left(\mathbb{F}_{q}\right)$ of $\mathbb{F}_{q}$-rational points of a simple algebraic group $G$ defined over a finite field $\mathbb{F}_{q}$.

Key words: unipotent representations, Schur indices, crystalline cohomology groups.
\end{abstract}

\section{Introduction}

Let $\mathbb{F}_{q}$ be a finite field with $q$ elements of characteristic $p$. Let $G$ be a connected, reductive linear algebraic group, defined over $\mathbb{F}_{q}$, with Frobenius map $F$, and let $G^{F}$ be the (finite) group of fixed points of $G$ by $F$. Then the problem of determining of the local Schur indices of the (complex) irreducible unipotent representations $\rho$ of $G^{F}$ can be reduced to the case where $G$ is a simple algebraic group of adjoint type and $\rho$ is cuspidal ([DL, Propositon 7.10], [Ge II, Remark 2.6], [Lu II, p. 28], [Ge I, Propositions 5.5, 5.6]).

Suppose that $G$ is a simple algebraic group of adjoint type and that $\rho$ is cuspidal unipotent representation of $G^{F}$. Then, in almost all cases, the local Schur indices of $\rho$ are determined by Lusztig [Lu V] and Geck [Ge I, II], more or less by a general method. However there are two remaining cases for which the above general method cannot be applied. They are the following: (i) the characters $E_{7}[ \pm \xi]$ in the group $G^{F}=E_{7}(q)$, where $q$ is an even power of $p$ such that $p \equiv 4(\bmod 4)$;

(ii) the characters $E_{8}[ \pm \sqrt{-1}]$ for $G^{F}=E_{8}(q)$ with $p=5$.

(see [Ge II]; as to te notations of characters of $G^{F}$, we follow those in [Ca, p. 483, p. 488].)

The first case was dealt with by Geck [Ge III], by investigating certain generalized Gelfand-Graev representations. For this, he has to assume that $p$ is large enough so that the result of Lusztig $[\mathrm{Lu} \mathrm{IV]}$ on generalized

2000 Mathematics Subject Classification : 12E, 14F, 16G, 20C, 20G. 
Gelfand-Graev representations can be applied (note that it relies on the general theory of Lie algebra, which requires that $p$ is not too small). Also it involves some explicit computations by using computer.

The idea for treating the second case was explained briefly in [Ge II] and was discussed in $\mathrm{Ph}$. D. thesis of Hezard [He]. It also use the generalized Gelfand-Graev representations of $E_{8}(q)$. Since $p=5$, the general theory of Lie algebra cannot be applied, and very delicate and precise computations are required.

In this paper, we with to propose a method of using crystalline cohomology groups in order to treat the above two cases. By realizing the above cuspidal unipotent representations $\rho$ on crystalline cohomology groups, it is possible to determine the $p$-local Schur index $m_{\mathbb{Q}_{p}}(\rho)$ of $\rho$. For any prime $\ell \neq p$, the $\ell$-local Schur index $m_{\mathbb{Q}_{p}}(\rho)$ of $\rho$ can be determined by making use of the realization of $\rho$ on the $\ell$-adic cohomology groups due to Lusztig $[\mathrm{Lu} \mathrm{V}]$. Thus the Schur index $m_{\mathbb{Q}_{p}}(\rho)$ of $\rho$ with respect to $\mathbb{Q}$ is determined by the Hasse principle. In particular, the argument works without restriction on $p$.

The method of making use of crystalline cohomology seems to be comparatively general since, by modifying our method, one can prove the following:

Theorem A Let $G$ be a simple algebraic group, defined over $\mathbb{F}_{q}$, with $\mathbb{F}_{q}$-rank $r$. Let $\rho$ by any cuspidal unipotent representation of $G^{F}$ with character $\chi_{\rho}$ and let $A\left(\rho, \mathbb{Q}_{p}\right)$ be the simple direct summand of the group algebra $\mathbb{Q}_{p}\left[G^{F}\right]$ associated with $\rho$. Let $\mathbb{Q}_{p}\left(\chi_{\rho}\right)=\mathbb{Q}_{p}\left(\chi_{\rho}\left(g_{0}\right), g_{0} \in G^{F}\right)$. Then the Hasse invariant of the simple algebra $A\left(\rho, \mathbb{Q}_{p}\right)$ (central over $\mathbb{Q}_{p}\left(\chi_{\rho}\right)$ ) can be given by $-(r / 2)\left[\mathbb{Q}_{p}\left(\chi_{\rho}\right): \mathbb{Q}_{p}\right]$.

Our result, with combining Lusztig's realization in the $\ell$-adic cohomology, can be interpreted in terms of motives over finite fields (see Milne [Mi II]).

Let $\rho$ be a cuspidal unipotent representation of $G^{F}$ ( $G$ simple). Let $w$ be a Weyl group element with minimal length $n=\ell(w)$ such that $\left(R^{1}(w), \rho\right)_{G^{F}} \neq 0$, where $R^{1}(w)$ is the Deligne-Lusztig virtual representation of $G^{F}$ associated with $w$ ([DL]). Let $\lambda q^{n \delta / 2}$ be the eigenvalue of Frobenius on $H^{n}\left(\bar{X}(w), \mathbb{Q}_{\ell}\right)$ associated with $\rho$ ([Lu II]; [DM, Théoremè 2.3, p. 48]), Here $\bar{X}(w)$ is the Hansen-Demazure-Deligne-Lusztig compactification of the Deligne-Lusztig variety $X(w)$ associated with $w$ ([DL, (9.10)]), 
$\overline{\mathbb{Q}_{\ell}}$ is an algebraic closure of the $\ell$-adic field $\mathbb{Q}_{\ell}(\ell \neq p), \delta$ is the minimal natural number such that $F^{\delta}$ acts trivially on the Weyl group of $G$, and $\lambda$ is a certain root of unity (cf. [DM]). Let $X$ be a simple motive with Weil $q^{\delta}$-number $q^{n \delta / 2}$ (uniquely determined up to isomorphisms; see [Mi II, p. 415]). Then

"Theorem B" Assume that Tate conjecture over finite fields holds (see $[$ Mi II] $]$. Then in the Brauer group of $\mathbb{Q}\left(\chi_{\rho}\right)$, the class of the simple direct summand $A(\rho, \mathbb{Q})$ of $\mathbb{Q}\left[G^{F}\right]$ associated with $\rho$ and the class of the endomorphism ring $\operatorname{End}(X)$ of $X$ are the same.

Theorem A also holds for cuspidal unipotent representations of the Suzuki and Ree groups ${ }^{2} B_{2}(q),{ }^{2} G_{2}(q),{ }^{2} F_{4}(q)$ except for the unique representation $\rho$ of ${ }^{2} F_{4}(q)$, such that $\left(R^{1}(w), \rho\right)_{G^{F}}$ is even for all $w$ ([Lu III, p. 375]); for this representation, the formula in Theorem A does not hold; $\rho$ has the property that $m_{\mathbb{Q}_{\ell}}(\rho)=1$ for $\ell \neq p$ and $m_{\mathbb{R}}(\rho)=m_{\mathbb{Q}_{p}}(\rho)=2$ (see [Ge I]).

\section{Notation}

$p$ is a fixed prime number and $k$ is an algebraic closure of the prime field of characteristic $p . q=p^{a^{\prime}}$ is a power of $p$ and $\mathbb{F}_{q}$ is the subfield of $k$ with $q$ elements. By a variety, we mean a separated reduced scheme of finite type over $k$ and we identify it with the set of its $k$-rational points.

If $\rho$ is an irreducible representation of a finite group $H$ over an algebraically closed field $C$ of characteristic 0 , then $\chi_{\rho}$ denotes its character and for a field $E$ of characteristic $0, E(\rho)=E\left(\chi_{\rho}\right)=E\left(\chi_{\rho}(h), h \in H\right)$ and $m_{E}(\rho)$ or $m_{E}\left(\chi_{\rho}\right)$ denotes the Schur index of $\rho$ with respect to $E$.

$\ell$ is any fixed prime number $\neq p$, and $\overline{\mathbb{Q}_{\ell}}$ is an algebraic closure of $\mathbb{Q}_{\ell}$. For a variety $X, H^{i}(X)$ (resp. $\left.H_{c}^{i}(X)\right)$ is the $i$-th étale cohomology group of $X$ (resp. the $i$-th étale cohomology of $X$ with compact supports) with coefficients in $\overline{\mathbb{Q}_{\ell}}$.

1.

Let $n$ be a positive integer, and let $\Lambda_{n}=\mathbb{Z} / \ell^{n} \mathbb{Z}$. Let $X, Y$ be varieties and let $f: X \rightarrow Y$ be a proper morphism. Then there exists a spectral sequence 


$$
\left(R_{c}^{i} \pi_{*}\right)\left(R^{j} f_{*}\right) F \Longrightarrow R_{c}^{i+j}(\pi f)_{*} F
$$

where $\pi: Y \rightarrow \operatorname{Spec}(k)$ is the structural morphism of $Y$ (see [Mi I, Theorem $3.2(\mathrm{c})$, p. 228]) (note that $R_{c}^{i} f_{*}=R^{j} f_{*}$ since $f$ is proper) and $F$ is any torsion (étale) sheaf of $\Lambda_{n}$-modules on $X$. Thus one of the edge homomorphisms of this spectral sequence gives $\Lambda_{n}$-homomorphisms ([CE, p. 329, Case B])

$$
H_{c}^{i}\left(Y, f_{*} F\right) \longrightarrow H_{c}^{i}(X, F) \quad(i \geqq 0) .
$$

(Note that $\left(R_{c}^{i} \pi_{*}\right)\left(R^{0} f_{*}\right) F=\left(R_{c}^{i} \pi_{*}\right) f_{*} F=H_{c}^{i}\left(Y, f_{*} F\right)$ and $R_{c}^{i}(\pi f)_{*} F=$ $H_{c}^{i}(X, F)$.)

Let $F^{\prime}$ be a torsion sheaf on $Y$ of $\Lambda_{n}$-modules, and let $F=f^{*} F^{\prime}$. Then, by composing the homomorphism $H_{c}^{i}\left(Y, F^{\prime}\right) \rightarrow H_{c}^{i}\left(Y, f_{*} f^{*} F^{\prime}\right)$ induced by the natural morphism $F^{\prime} \rightarrow f_{*} f^{*} F^{\prime}$ with the homomorphism $(*)$, we get a $\Lambda_{n}$-homomorphism $H_{c}^{i}\left(Y, F^{\prime}\right) \rightarrow H_{c}^{i}\left(X, f^{*} F^{\prime}\right)$. By letting $F^{\prime}=\Lambda_{n}$, and by using the canonical isomorphism $f^{*} \Lambda_{n} \stackrel{\sim}{\rightarrow} \Lambda_{n}$, we get a $\Lambda_{n}$-homomorphism

$$
f_{n}^{*}: H_{c}^{i}\left(Y, \Lambda_{n}\right) \longrightarrow H_{c}^{i}\left(X, \Lambda_{n}\right) \quad(i \geqq 0) .
$$

We note that if $Z$ is a variety and $g: Y \rightarrow Z$ is a proper morphism, then we have

$$
(* * *) \quad(g f)_{n}^{*}=f_{n}^{*} g_{n}^{*} .
$$

Assume that $X, Y$ are proper over $\operatorname{Spec}(k)$. Then, by the functoriality ([Sri. p. 41]), we get a natural $\Lambda_{n}$-homomorphism $H^{i}\left(Y, F^{\prime}\right) \rightarrow H^{i}\left(X, f^{*} F^{\prime}\right)$, which, as we can check, coincides with the above homomorphism $H^{i}\left(Y, F^{\prime}\right) \rightarrow H^{i}\left(Y, f_{*} f^{*} F^{\prime}\right) \rightarrow H^{i}\left(X, f^{*} F^{\prime}\right)$.

Returning to the general case with $f: X \rightarrow Y$ proper, let $\psi_{n}$ : $H_{c}^{i}\left(Y, \Lambda_{n+1}\right) \rightarrow H_{c}^{i}\left(Y, \Lambda_{n}\right), \phi_{n}: H_{c}^{i}\left(X, \Lambda_{n+1}\right) \rightarrow H_{c}^{i}\left(X, \Lambda_{n}\right)$ be homomorphisms which are induced by the natural morphism $\Lambda_{n+1} \rightarrow \Lambda_{n}$. Then we have $\phi_{n} f_{n+1}^{*}=f_{n}^{*} \psi_{n}$. Hence, by taking projective limits, we get a $\mathbb{Z}_{\ell}$-homomorphism

$$
\varliminf_{n} f_{n}^{*}: H_{c}^{i}\left(Y, \mathbb{Z}_{\ell}\right)={\underset{\lim }{n}}_{n} H_{c}^{i}\left(Y, \Lambda_{n}\right) \longrightarrow H_{c}^{i}\left(X, \mathbb{Z}_{\ell}\right) .
$$

By tensoring with $\mathbb{Q}_{\ell}$, we get $\mathbb{Q}_{\ell}$-linear maps

$$
H_{c}^{i}\left(Y, \mathbb{Q}_{\ell}\right) \longrightarrow H_{c}^{i}\left(X, \mathbb{Q}_{\ell}\right) \quad(i \geqq 0),
$$


hence we get $\overline{\mathbb{Q}_{\ell}}$-linear maps

$$
f^{*}: H_{c}^{i}(Y) \longrightarrow H_{c}^{i}(X) \quad(i \geqq 0) .
$$

We note that, if $g: Y \rightarrow Z$ is proper, then

$$
(g f)^{*}=f^{*} g^{*} \text {. }
$$

Now let $G$ be a connected, reductive linear algebraic group over $k$, defined over $\mathbb{F}_{q}$, with Frobenius map $F$. Let $X_{G}$ be the projective variety of all Borel subgroups of $G$. Let $F: X_{G} \rightarrow X_{G}$ be the map defined by $B \rightarrow$ $F(B)$, which is the Frobenius map corresponding to the natural $\mathbb{F}_{q}$-rational structure of $X_{G}$. $G$ acts on $X_{G}$ by the conjugations: $B \rightarrow g B g^{-1}, g \in G$, $B \in X_{G}$.

For the sake of later use, let me allow to explain this action of $G$ on $X_{G}$. Let $k[G]$ be the $k$-algebra of regular functions on $G$. Then $G$ acts on it by $(h \cdot g)(x)=h\left(x g^{-1}\right), g \in G, h \in k[G], x \in G$. Then there is a finitedimensional, $G$-stable subspace $V$ of $k[G]$ and a line $L$ through $0(\subset V)$ such that $B^{*}=\{g \in G \mid g(L)=L\}$, where $B^{*}$ is a previously fixed $F$-stable Borel subgroup of $G$. Let $\mathbb{P}(V)$ be the projective space associated with $V$ and let $[L]$ be the class of $L$ in $\mathbb{P}(V)$. Note that $V$ and $L$ can be chosen so that they are defined over $\mathbb{F}_{q}$. The homogeneous space $G / B^{*}$ is defined to be the orbit $G \cdot[L]$ in $\mathbb{P}(V)$. Since $G / B^{*}$ is projective, it is complete, hence $G / B^{*}=G \cdot[L]$ is closed in $\mathbb{P}(V)$. Let $\rho: G \rightarrow \mathrm{GL}(V)$ be the representation which is determined by the $G$-module $V$. Then, for each $g \in G, \rho(g)$ is an automorphism of the affine space $V$, which hence induces a $k$-algebra automorphism $\theta(g)$ of $k[V]$. With respect to a basis of the $\mathbb{F}_{q}$-structure $V_{0}$ of $V, k[V]$ can be viewed naturally as a polynomial ring $k\left[T_{0}, \ldots, T_{d}\right]$ over $k$ $\left(d+1=\operatorname{dim}_{\mathbb{F}_{q}}\left(V_{0}\right)\right)$. Then, for $g \in G, \theta(g)$ is a homogeneous automorphism of $k\left[T_{0}, \ldots, T_{d}\right]$ of degree 0 , so that it induces a ring automorphism $\bar{\theta}(g)$ of $k\left[G / B^{*}\right]=k[G \cdot[L]]$. Then it is well known that, for $g \in G$, the automorphism $\bar{\theta}(g)$ induces an automorphism of $G / B^{*}$, which coincides with the mapping $h B^{*} \rightarrow g h B^{*}, h B^{*} \in G / B^{*}$. Since $X_{G}$ is isomorphic to $G / B^{*}$ naturally, the adjoint action of $g \in G$ on $X_{G}$ is induced by a $k$-algebra automorphism of a $k$-algebra $A$ such that $X_{G}=\operatorname{Proj}(A)$.

Now, we let $G$ act on $X_{G} \times X_{G}$ diagonally. Then $W_{G}=G \backslash\left(X_{G} \times X_{G}\right)$ has a natural group structure, which is called the Weyl group of $G$ ([DL, 1.2], [Lu I, (1.2)]). For $w \in W_{G}$, let $X(w)=\left\{B \in X_{G} \mid(B, F(B)) \in w\right\}$. Then $X(w)$ is a locally closed smooth subvariety of $X_{G}$, purely of dimension $\ell(w)$, 
where $\ell()$ is the length function on $W_{G}([\mathrm{DL}, 1.4])$. For $g_{0} \in G^{F}, X(w)$ is $g_{0}$-stable, so, for each $i \geqq 0$, we have an automorphism $g_{0}^{*}$ of $H_{c}^{i}(X(w))$. We consider $H_{c}^{i}(X(w))$ as $G^{F}$-modules by $\left(g_{0}^{-1}\right)^{*}, g_{0} \in G^{F}$. For $i \geqq 0$, $H_{c}^{i}(X(w))$ is a $\overline{\mathbb{Q}}_{\ell}\left[G^{F}\right]$-module with $\mathbb{Q}_{\ell}$-structure $H_{c}^{i}\left(X(w), \mathbb{Q}_{\ell}\right)$.

Let $\delta$ be the minimal positive integer such that $F^{\delta}$ acts trivially on $W_{G}$. Then, for $w \in W_{G}, X(w)$ is $F^{\delta}$-stable. Let $w \in W_{G}$. Then the morphism $F^{\delta}: X(w) \rightarrow X(w)$ is finite, hence proper, so, for $i \geqq 0, F^{\delta}$ induces a $\overline{\mathbb{Q}} \ell^{-}$linear map $\left(F^{\delta}\right)^{*}: H_{c}^{i}(X(w)) \rightarrow H_{c}^{i}(X(w))$.

Let $M$ be a simple $\overline{\mathbb{Q}_{\ell}}\left[G^{F}\right]$-module. Then we say that $M$ has depth $t$ if there is an $F$-stable subset $I$ of the set $S$ of simple reflections in $W_{G}$ with $\left|I_{F}\right|=r-t$, where $I_{F}$ is the set of orbits of $F$ on $I$ and $r$ is the semisimple $\mathbb{F}_{q}$-rank of $G$, such that, for an $F$-stable parabolic subgroup $P_{I}$ of $G$ corresponding to $I$, with unipotent radical $U_{I}, M^{U_{I}^{F}}$ contains a nonzero cuspidal $L^{F}$-module, where $L=P_{I} / U_{I}$ and $M^{U_{I}^{F}}$ is the subspace of $M$ consisting of elements of $M$ fixed by $U_{I}^{F}$ (see [Lu I, §4]).

Let $M$ be any (finitely generated) $\overline{\mathbb{Q}_{\ell}}\left[G^{F}\right]$-module For an integer $t \geqq 0$, let $M^{(t)}$ be the subspace of $M$ defined as the sum of all simple $\overline{\mathbb{Q}_{\ell}}\left[G^{F}\right]$-submodules of $M$ of depth $t$. Then we have $M=\bigoplus_{t \geqq 0} M^{(t)} \cdot M^{(0)}$ is the cuspidal part of $M$.

Now assume that $G$ is a simple algebraic group of type $\left(E_{7}\right)$. Let $s_{1}, \ldots, s_{7}$ be the simple reflections in $W_{G}$. Put $c=s_{1} \cdots s_{7}$ and $f=$ $\left(s_{1}, \ldots, s_{7}\right)$. Let $X_{f}=X(c)$. Then $X_{f}$ is a smooth affine irreducible subvariety of dimension 7 ([Lu I, (2.8), (4.8)]). We have $H_{c}^{i}\left(X_{f}\right)^{(0)}=0$ for $i \neq 7$ and $H_{c}^{7}\left(X_{f}\right)^{(0)}=H_{c}^{7}\left(X_{f}\right)_{\sqrt{-q^{7}}} \oplus H_{c}^{7}\left(X_{f}\right)_{-\sqrt{-q^{7}}}$, where $H_{c}^{7}\left(X_{f}\right)_{\sqrt{-q^{7}}}$ (resp. $H_{c}^{7}\left(X_{f}\right)_{-\sqrt{-q^{7}}}$ ) is the subspace of $H_{c}^{7}\left(X_{f}\right)$ on which $F^{*}$ acts by multiplication by $\sqrt{-q^{7}}$ (resp. $\left.-\sqrt{-q^{7}}\right)([\mathrm{Lu} \mathrm{I},(6.1),(7.1),(7.3),(7.4)(\mathrm{c})])$. They afford two non-isomorphic cuspidal unipotent representations of $G^{F}$ over $\overline{\mathbb{Q}_{\ell}}$ (see [Lu III, pp. 364-5], Cater [Ca, pp. 482-3]). And they are all the cuspidal unipotent representations of $G^{F}$.

Let $\rho$ be a (complex) cuspidal unipotent representation of $G^{F}$. Then $\mathbb{Q}\left(\chi_{\rho}\right)=\mathbb{Q}\left(\sqrt{-q^{7}}\right)$ (cf. [Ge I, p. 21]). Since $\chi_{\rho}$ is not real, we have $m_{\mathbb{Q}_{\infty}}(\rho)=1$. Let $\tau: \mathbb{C} \sim \overline{\mathbb{Q}_{\ell}}$ be an isomorphism. Then, since $\left(H_{c}^{7}\left(X_{f}\right), \rho^{\tau}\right)_{G^{F}}$ $=1$ and $H_{c}^{7}\left(X_{f}\right)$ is defined over $\mathbb{Q}_{\ell}$, by a property of the Schur index, we have $m_{\mathbb{Q}_{\ell}}(\rho)=1$. Since $\ell$ is any prime number $\neq p$, by Hasse's sum formula, we must have $m_{\mathbb{Q}_{p}}(\rho)=1$ (hence $m_{\mathbb{Q}}(\rho)=1$ ) if the number of the places of $\mathbb{Q}\left(\chi_{\rho}\right)$ lying above $p$ is equal to one, and this is the case unless $q$ is an even 
power of $p$ such that $p \equiv 1(\bmod 4)$.

To treat the remaining case we use crystalline cohomology. To do so we need some analysis of Lusztig's results in [Lu I].

Let $X_{f}^{\cdot}=\left\{\left(B_{0}, B_{1}, \ldots, B_{7}\right) \in X_{G}^{8} \mid\left(B_{i-1}, B_{i}\right) \in s_{i} \cup e\right.$ for $1 \leqq i \leqq$ 7 , and $\left.F\left(B_{0}\right)=B_{7}\right\}$. Then $X_{f}$ is a smooth projective variety and $X_{f}$ can be identified with the open dense subvariety $\left\{\left(B_{0}, B_{1}, \ldots, B_{7}\right) \in X_{f} \mid B_{0} \neq\right.$ $\left.B_{1} \neq \cdots \neq B_{7}\right\}$ of $X_{f}$ (by Bruhat lemma) ([DL, 9.10]). Let $F: X_{G}^{8} \rightarrow$ $X_{G}^{8}:\left(B_{0}, B_{1}, \ldots, B_{7}\right) \rightarrow\left(F\left(B_{0}\right), F\left(B_{1}\right), \ldots, F\left(B_{7}\right)\right)$. Then $X_{f}$ is $F$-stable and $G^{F}$ acts on it diagonally. The inclusion $X_{f} \hookrightarrow X_{f}$ is $F-G^{F}$-equivariant. Then this inclusion map induces an isomorphism $H_{c}^{i}\left(X_{f}\right)^{(0)} \stackrel{\sim}{\rightarrow} H_{c}^{i}\left(X_{f}\right)^{(0)}$ $(i \geqq 0)([\mathrm{Lu} \mathrm{I},(4.3 .1)])$.

Lemma 1 ([Lu I, §4]) We have $H^{7}\left(X_{f}^{\cdot}\right)=H^{7}\left(X_{f}^{\cdot}\right)^{(0)}$.

In fact, let me allow to use the notations of [Lu I, §4] freely.

The exact sequence (4.2.3) of [Lu I, $\S 4$ ] for $a=7$ and $i=7$ can be read:

$$
\cdots \longrightarrow H_{c}^{7}\left(X_{f}\right)^{(t)} \stackrel{\alpha^{(t)}}{\longrightarrow} H_{c}^{7}\left(X_{f}\right)^{(t)} \stackrel{\beta^{(t)}}{\longrightarrow} H_{c}^{8}\left(D_{6}\right)^{(t)} \longrightarrow \cdots .
$$

We see from the table on page 146 of $[\mathrm{Lu} \mathrm{I}]$ that the absolute value of each eigenvalue of $F^{*}$ on $H_{c}^{7}\left(X_{f}\right)$ other than $\pm \sqrt{-q^{7}}$ is an integral power of $q$. On the other hand, we know from Deligne's theorem on the eigenvalues of Frobenius [De] that the absolute value of any eigenvalue of $F^{*}$ on $H^{7}\left(X_{f}^{\cdot}\right)$ is $q^{7 / 2}$. Since the actions of $F$ and $G^{F}$ commute, we see that $\alpha^{(t)}=0$ for $t \geqq 1$.

Next we show that $\beta^{(t)}=0$ for all $t \geqq 0$, which would imply the desired assertion.

Assume that $t \geqq 2$. Then, by the statement on page 122 , lines $7-8$, of [Lu I], we see that $H^{7}\left(D_{6}\right)^{(t)}$ is isomorphic as $G^{F}$-modules to a quotient of $\bigoplus H_{c}^{7}\left(X_{f}^{\cdot}(I)\right)$. Moreover, by a standard argument from linear algebra by $|I| \leqq 6$

using the exact sequences in lines 5,6 on page 122 in [Lu I], that the set of eigenvalues of $F^{*}$ on $H^{7}\left(D_{6}\right)^{(t)}$ is contained in the set of eigenvalues of $F^{*}$ on $\bigoplus_{|I| \leq 6} H_{c}^{7}\left(X_{f}^{\cdot}(I)\right)$. By (4.2.1) of [Lu I, p. 119], by the Künneth formula, and by the table on page 146 of [Lu I, p. 119], we see that the absolute value of each eigenvalue of $F^{*}$ on $\bigoplus_{|I| \leqq 6} H_{c}^{7}\left(X_{f}(I)\right)$, hence on $H^{7}\left(D_{6}\right)^{(t)}$, is an integral power of $q$. Thus $\beta^{(t)}=0$ for $t \geqq 2$. by the formula on page 121 , 
line 13 , of $[\mathrm{Lu} \mathrm{I}]$, we see that $H^{7}\left(D_{6}\right)^{(1)}=0$. And, by the formula on page 120 , line 15 , of $[\mathrm{Lu} \mathrm{I}]$, we have $H^{7}\left(D_{6}\right)^{(0)}=0$. Thus $\beta^{(t)}=0$ for all $t \geqq 0$.

2.

Let $W\left(\mathbb{F}_{q}\right)$ be the ring of Witt vectors of $\mathbb{F}_{q}$ and let $K$ be its quotient field. Let $\sigma$ be the Frobenius automorphism of $W\left(\mathbb{F}_{q}\right)$ (induced by the automorphism $x \rightarrow x^{p}$ of $\mathbb{F}_{q}$ ); we also denote by $\sigma$ its extension to $K$. For a proper smooth scheme $X_{0}$ over $\mathbb{F}_{q}$, let $H^{i}\left(X_{0} / W\left(\mathbb{F}_{q}\right)\right)$ be the $i$-th crystalline cohomology group of $X_{0}$ over $W\left(\mathbb{F}_{q}\right)$ (see Berthelot [Ber, p. 525]; also see Illusite [Ill, 1.2, p. 44]), and let $H_{\text {crys }}^{i}\left(X_{0}\right)=H^{i}\left(X_{0} / W\left(\mathbb{F}_{q}\right)\right) \otimes_{W\left(\mathbb{F}_{q}\right)} K$.

Let $n$ be a positive integer, and let $W_{n}=W\left(\mathbb{F}_{q}\right) / p^{n} W\left(\mathbb{F}_{q}\right)\left(W_{1}=\mathbb{F}_{q}\right)$. Let $g: X_{0} \rightarrow Y_{0}$ be a morphism of proper smooth schemes $X_{0}, Y_{0}$ over $\mathbb{F}_{q}$, and suppose that the diagram

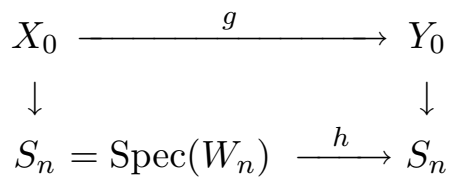

commutes. Here, $X_{0} \rightarrow S_{n}$ is the composition: $X_{0} \rightarrow \operatorname{Spec}\left(\mathbb{F}_{q}\right)=$ $\operatorname{Spec}\left(W_{1}\right) \rightarrow S_{n}\left(Y_{n} \rightarrow S_{n}\right.$ is defined similarly) and $h$ is a PD morphism (see [Ber, p. 30] or [BO, p. 3.1]). Then we have a morphism of topoi:

$$
g_{\text {cris }}=\left(g_{\text {cris }}^{*}, g_{\text {cris* }}\right):\left(X_{0} / S_{n}\right)_{\text {cris }} \longrightarrow\left(Y_{0} / S_{n}\right)_{\text {cris }}
$$

(see [Ber, Théorème 2.2.3, p. 197] or [BO, p. 5.3, p. 5.16]). Here $\left(X_{0} / S_{n}\right)_{\text {cris }}$ is the topos of sheaves on the site $\operatorname{Cris}\left(X_{0} / S_{n}\right)$ (see [Ber, p. 180] or [BO, p. 5.3]) $\left(\left(Y_{0} / S_{n}\right)_{\text {cris }}\right.$ is defined similarly). Let $O_{X_{0} / S_{n}}$ (resp. $\left.O_{Y_{0} / S_{n}}\right)$ be the "structural sheaf" of $X_{0}$ over $S_{n}$ (resp. $Y_{0}$ over $S_{n}$ ) ([Ber, p. 183] or [BO, p. 5.4]; also cf. [Ill, p. 44]). Then, by the functoriality (or by the spectral sequence in [BO, p. 5.16]), there is a natural map $H^{i}\left(\mathrm{Cris}\left(Y_{0} / S_{n}\right), O_{Y_{0} / S_{n}}\right) \rightarrow$ $H^{i}\left(\operatorname{Cris}\left(X_{0} / S_{n}\right), g_{\text {cris }}^{*} O_{X_{0} / S_{n}}\right)$ for each $i$, where $H^{i}\left(\operatorname{Cris}\left(Y_{0} / S_{n}\right), O_{Y_{0} / S_{n}}\right)$ is the $i$-th cohomology group of the site $\operatorname{Cris}\left(Y_{0} / S_{n}\right)$ with coefficients in $O_{Y_{0} / S_{n}}\left([\mathrm{Ber}\right.$, p. 180, p. 184] $)\left(H^{i}\left(\mathrm{Cris}\left(X_{0} / S_{n}\right), g_{\text {cris }}^{*} O_{X_{0} / S_{n}}\right)\right.$ is defined similarly). By composing this map with the natural map $H^{i}\left(\operatorname{Cris}\left(X_{0} / S_{n}\right), g_{\text {cris }}^{*} O_{Y_{0} / S_{n}}\right) \rightarrow H^{i}\left(\operatorname{Cris}\left(X_{0} / S_{n}\right), O_{X_{0} / S_{n}}\right)$ induced by the natural morphism $g_{\text {cris }}^{*} O_{Y_{0} / S_{n}} \rightarrow O_{X_{0} / S_{n}}$ (see [Ber, (2.2.4), p. 199]), we get a map 


$$
g_{n}^{*}: H^{i}\left(\operatorname{Cris}\left(Y_{0} / S_{n}\right), O_{Y_{0} / S_{n}}\right) \longrightarrow H^{i}\left(\operatorname{Cris}\left(X_{0} / S_{n}\right), O_{X_{0} / S_{n}}\right) .
$$

If

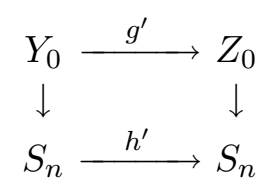

is another commutative diagram, where $Z_{0}$ is a proper smooth variety over $\mathbb{F}_{q}$ and $h^{\prime}$ is a PD morphism, we have

$$
\left(g^{\prime} g\right)_{n}^{*}=\left(g_{n}^{*}\right)\left(g_{n}^{\prime *}\right)
$$

(cf. [Ber, Proposition 2.2.6, p. 200]).

We also have natural maps $p_{n}: H^{i}\left(\mathrm{Cris}\left(X_{0} / S_{n+1}\right), O_{X_{0} / S_{n+1}}\right) \rightarrow$ $H^{i}\left(\operatorname{Cris}\left(X_{0} / S_{n}\right), O_{X_{0} / S_{n}}\right), \quad q_{n}: H^{i}\left(\operatorname{Cris}\left(Y_{0} / S_{n+1}\right), O_{Y_{0} / S_{n+1}}\right) \rightarrow$ $H^{i}\left(\operatorname{Cris}\left(Y_{0} / S_{n}\right), O_{Y_{0} / S_{n}}\right)$, and we have $p_{n} g_{n+1}^{*}=g_{n}^{*} q_{n}$. Therefore, by taking projective limits, we get a map

$$
g^{*}:=\lim _{n} g_{n}^{*}: H^{i}\left(Y_{0} / W\left(\mathbb{F}_{q}\right)\right) \longrightarrow H^{i}\left(X_{0} / W\left(\mathbb{F}_{q}\right)\right) \quad(i \geqq 0) .
$$

We have $\left(g^{\prime} g\right)^{*}=g^{*} g^{\prime *}$.

Let $X_{0}$ be a projective smooth scheme over $\mathbb{F}_{q}$. Let $F_{\text {abs }}: X_{0} \rightarrow X_{0}$ be the absolute Frobenius endomorphism of $X_{0}: F_{\text {abs }}$ is the identity map on the underlying space of $X_{0}$ and, for each section $h$ in the structural sheaf $O_{X_{0}}$ of $X_{0}$, we have $F_{\text {abs }}(h)=h^{p}$. Then we have a commutative diagram

$$
\begin{array}{cc}
X_{0} \stackrel{F_{\text {abs }}}{\longrightarrow} & X_{0} \\
\downarrow & \\
S_{n} \stackrel{\downarrow}{h_{n}} & S_{n},
\end{array}
$$

where $h_{n}$ is the PD morphism induced by $\sigma: W_{n} \rightarrow W_{n}$. Then we have a $\sigma$-linear endomorphism $\left(F_{\text {abs }}\right)^{*}$ of $H^{i}\left(X_{0} / W\left(\mathbb{F}_{q}\right)\right)$ for each $i$. Hence we get a $\sigma$-linear endomorphism $\phi=\left(F_{\text {abs }}\right)^{*} \otimes \sigma$ of $H_{\text {crys }}^{i}\left(X_{0}\right)$ for each $i$. This makes each $\left(H_{\text {crys }}^{i}\left(X_{0}\right), \phi\right)$ an isocrystal over $K$, i.e. a finite-dimensional vector space over $K$ with $\sigma$-linear bijection.

Let $X_{0}$ be as above. Recall that $q=p^{a^{\prime}}$. Then $F_{0}=\left(F_{\text {abs }}\right)^{a^{\prime}}$ is the Frobenius endomorphism of $X_{0}$; if $X=X_{0} \times_{\mathbb{F}_{q}} k$, then $F=F_{0} \times 1$ is the Frobenius endomorphism of $X$ which corresponds to the $\mathbb{F}_{q}$-rational structure $X_{0}$ on $X$. 
Let $X=\operatorname{Proj}(A), Y=\operatorname{Proj}(B)$ be two projective varieties defined over $\mathbb{F}_{q}$, and let $g$ and $h$ be automorphisms of $X$ and $Y$ respectively, defined over $\mathbb{F}_{q}$; Assume that $g$ (resp. $h$ ) is the restriction to $X$ (resp. $Y$ ) of an automorphism of an ambient projective space, with the standard $\mathbb{F}_{q}$-rational structure, defined over $\mathbb{F}_{q}$. Then we see that $g \times h$ is the automorphism of $X \times X$ which is induced by a $k$-algebra automorphism of $A \otimes_{k} B$.

Now let the assumptions and the notations be as in $\S 1$. Recall that $X_{f}$ is an $F$-stable closed subvariety of $X_{G}^{8}$. Suppose that $X_{f}^{\cdot}=\operatorname{Proj}(A)$. Then, for each $g_{0} \in G^{F}$, the automorphism $g_{0}: X_{f} \rightarrow X_{f}^{*}$ is induced by a $k$-algebra automorphism $\theta\left(g_{0}\right)$ of $A$ which is homogeneous of degree 0 . Let

$$
A_{0}=\left\{x \in A \mid F(x)=x^{q}\right\} .
$$

Then $X_{f, 0}=\operatorname{Proj}\left(A_{0}\right)$ is the $\mathbb{F}_{q}$-rational structure on $X_{f}$ determined by $F: X_{f} \rightarrow X_{f}$. Let $g_{0} \in G^{F}$. Then, since $\theta\left(g_{0}\right): A \rightarrow A$ is a ring automorphism, for $x \in A_{0}$, we have

$$
F\left(\theta\left(g_{0}\right)(x)\right)=\theta\left(g_{0}\right)(F(x))=\theta\left(g_{0}\right)\left(x^{q}\right)=\theta\left(g_{0}\right)(x)^{q},
$$

so $\theta\left(g_{0}\right)(x) \in A_{0}$. So $\theta\left(g_{0}\right)$ induces a ring automorphism of $A_{0}$, hence induces an endomorphism $g_{0}$ of $X_{f, 0}$. Thus we get an endomorphism $\left(g_{0}\right)^{*}$ of $H^{i}\left(X_{f, 0} / W\left(\mathbb{F}_{q}\right)\right)$ for $i \geqq 0$. It is clear that $\left(h_{0} g_{0}\right)^{*}=\left(g_{0}\right)^{*}\left(h_{0}\right)^{*}\left(h_{0} \in G^{F}\right)$. Thus each $H^{i}\left(X_{f, 0} / W\left(\mathbb{F}_{q}\right)\right)$ is a $G^{F}$-module by the actions $\left(g_{0}^{-1}\right)^{*}$, $g_{0} \in G^{F}$.

Let $g_{0} \in G^{F}$. Then the graph of $g_{0}: X_{f, 0} \rightarrow X_{f, 0}$ defines a cycle in $X_{f, 0} \times_{S_{n}} X_{f, 0}$ of codimension $7(n \geqq 1)$, hence, by the Künneth formula and the Poincarè duality theorem for crystalline cohomology, its class in $H^{14}\left(\left(X_{f, 0}^{\cdot} \times_{S_{n}} X_{f, 0}^{\cdot}\right) / S_{n}, O_{\left(X_{f, 0} \times S_{n} X_{f, 0}\right) / S_{n}}\right)$, hence in $H_{\text {crys }}^{14}\left(X_{f, 0}^{\cdot} \times_{\mathbb{F}_{q}} X_{f, 0}^{\cdot}\right)$ determines a linear endomorphism of $H_{\mathrm{crys}}^{1}\left(X_{f, 0}\right)$ for each $i$, which is just $\left(g_{0}\right)^{*} \otimes 1$ (cf. Kleimann [Kl, 3, pp. 11-2] and Berthelot [Ber, Chap. VII, $\S 3$, Lemma 3.1.4, p. 575]). Similar statements also hold for étale cohomology (cf. [Mi I, Chap. VI, §12, Lemma 12.1]). Thus by Theorem 2 of Katz and Messing $[\mathrm{KM}]$, we see that, for each $i$, the characteristic polynomial of $\left(g_{0}\right)^{*} \otimes 1$ on $H_{\text {crys }}^{i}\left(X_{f, 0}^{\cdot}\right)$ coincides with the characteristic polynomial of $\left(g_{0}\right)^{*}$ on $H^{i}\left(X_{f}\right)$ (they have coefficients in $\mathbb{Z}$ ). In particular, we have

$$
\operatorname{Tr}\left(\left(g_{0}\right)^{*} \otimes 1, H_{\text {crys }}^{i}\left(X_{f, 0}^{\cdot}\right)\right)=\operatorname{Tr}\left(\left(g_{0}\right)^{*}, H^{i}\left(X_{f}^{\cdot}\right)\right) \quad(i \geqq 0) .
$$


(This argument was inspired by Lusztig [Lu I, p. 121, line 24].) We also see that, by Theorem 1 of $[\mathrm{KM}]$, the eigenvalues of $\left(F_{0}\right)^{*} \otimes 1$ on $H_{\text {crys }}^{7}\left(X_{f, 0}^{\cdot}\right)$ coinside the eigenvalue of $F^{*}$ on $H^{7}\left(X_{f}^{*}\right)$.

Lemma 2 Let $g_{0} \in G^{F}$. Then $F_{\text {abs }} g_{0}=g_{0} F_{\text {abs }}$ on the scheme $X_{f, 0}$. Thus $\phi\left(\left(g_{0}\right)^{*} \otimes 1\right)=\left(\left(g_{0}\right)^{*} \otimes 1\right) \phi$ on $H_{\text {crys }}^{i}\left(X_{f, 0}\right)(i \geqq 0)$ (recall that $\phi=$ $\left.\left(F_{\text {abs }}\right)^{*} \otimes \sigma\right)$.

In fact, on the underlying space of $X_{f, 0}, F_{\text {abs }}$ is the identity. For $x \in A_{0}$, we have $\theta\left(g_{0}\right)\left(F_{\text {abs }}(x)\right)=\theta\left(g_{0}\right)\left(x^{p}\right)=\theta\left(g_{0}\right)(x)^{p}=F_{\text {abs }}\left(\theta\left(g_{0}\right)(x)\right)$. The last assertion is clear.

Assume that $q$ is an even power of $p$ such that $p \equiv 1(\bmod 4)$. Then we have $\sqrt{-q^{7}} \in \mathbb{Q}_{p}$. The eigenvalues of $\left(F_{0}\right)^{*} \otimes 1=\phi^{a^{\prime}}\left(q=p^{a^{\prime}}\right)$ on $H_{\text {crys }}^{7}\left(X_{f, 0}\right)$ are $\pm \sqrt{-q^{7}}$. Let $M_{+}$(resp. $M_{-}$) be the generalized $\sqrt{-q^{7}}$-eigenspace (resp. $-\sqrt{-q^{7}}$-eigenspace) of $H_{\text {crys }}^{7}\left(X_{f, 0}\right)$. Then, since the action of $G^{F}$ and $\left(F_{0}\right)^{*} \otimes 1$ on $H_{\text {crys }}^{7}\left(X_{f, 0}^{\cdot}\right)$ commute, we see that $M_{+}$and $M_{-}$are $G^{F_{-}}$ submodules of $H_{\text {crys }}^{7}\left(X_{f, 0}^{\cdot}\right)$. Hence, by (1), we see that they are absolutely irreducible $G^{F}$-modules over $K$ and $H_{\text {crys }}^{7}\left(X_{f, 0}^{\cdot}\right)=M_{+} \oplus M_{-}$; moreover we see that the actions of $\left(F_{0}\right)^{*} \otimes 1$ on $M_{+}$and $M_{-}$are semisimple.

By Lemma 2, we see that $\phi\left(M_{+}\right)$is a $G^{F}$-module. For $g_{0} \in G^{F}$, we have

$$
\operatorname{Tr}\left(\left(g_{0}\right)^{*} \otimes 1, \phi\left(M_{+}\right)\right)=\sigma\left(\operatorname{Tr}\left(\left(g_{0}\right)^{*} \otimes 1, M_{+}\right)\right)=\operatorname{Tr}\left(\left(g_{0}\right)^{*} \otimes 1, M_{+}\right)
$$

since $\mathbb{Q}_{p}\left(\chi_{\rho}\right)=\mathbb{Q}_{p}\left(\sqrt{-q^{7}}\right)=\mathbb{Q}_{p}\left(\rho\right.$ is the representation afforded by $\left.M_{+}\right)$ (Geck [Ge I, §5]). So $\phi\left(M_{+}\right)$is isomorphic to $M_{+}$as $G^{F}$-modules. Since $M_{+}$and $M_{-}$are not isomorphic, we must have $\phi\left(M_{+}\right)=M_{+}$. Similarly, we must have $\phi\left(M_{-}\right)=M_{-}$. Therefore $\left(M_{+}, \phi\right)$ and $\left(M_{-}, \phi\right)$ are semisimple isocrystals over $K$ (cf. Milne [Mi II, Proposition 2.10, p. 417]).

Let $M$ be $M_{+}$or $M_{-}$, and let

$$
U: K\left[G^{F}\right] \longrightarrow \operatorname{End}_{K}(M)
$$

be the corresponding representation of $K\left[G^{F}\right]$. Since $U$ is absolutely irreducible, we have $U\left(K\left[G^{F}\right]\right)=\operatorname{End}_{K}(M) \cong M_{d}(K)$, where $M_{d}(K)$ is the $K$-algebra of all $d \times d$-matrices over $K$ with $d=\operatorname{dim}_{K} M$. Let $B=U\left(\mathbb{Q}_{p}\left[G^{F}\right]\right)$. Then there is a division algebra $D$, central over $\mathbb{Q}_{p}=$ $\mathbb{Q}_{p}\left(\sqrt{-q^{7}}\right)$, such that $B \cong M_{n}(D)$, where if $m$ denotes the index of $D$, then $d=n m$ (cf. Curtis and Reiner [CR, p. 468]). We note that $m=$ 
$m_{\mathbb{Q}_{p}}(U)$. We have $B \otimes_{\mathbb{Q}_{p}} K \simeq M_{d}(K) \simeq \operatorname{End}_{K}(M)$ ([loc. cit.]).

Let

$$
\operatorname{End}(M, \phi)=\left\{h \in \operatorname{End}_{K}(M) \mid \phi h=h \phi\right\} .
$$

This is a $\mathbb{Q}_{p}$-form of the centralizer $Z_{\operatorname{End}_{K}(M)}\left(\pi_{M}\right)$ of $\pi_{M}=\phi^{a^{\prime}}$ in $\operatorname{End}_{K}(M)$ (see Kottwitz [Ko, p. 410]; also see Milne [Mi II, p. 417]). By Lemma 2, we see that $B$ is contained in $\operatorname{End}(M, \phi)$. But,, as $B \otimes_{\mathbb{Q}_{p}} K=\operatorname{End}_{K}(M)$, we must have $B=\operatorname{End}(M, \phi)$. Therefore, as $(M, \phi)$ is semisimple, there is a simple subisocrystal $(X, \phi)$ of $(M, \phi)$ such that $\operatorname{End}(X, \phi) \cong D$. By Lemma 11.3 of $[\mathrm{Ko}]$ (also see [Mi II, Proposition 2.14]), we see that the Hasse invariant of $D$ is $1 / 2$. Therefore $m_{\mathbb{Q}_{p}}(U)=2$.

We note that $G^{F}=E_{7}(q)$ has just two isomorphism classes of cuspidal unipotent representations.

The following theorem is due to Geck [Ge III] except for (ii) where he had to assume that $p$ is large enough. Our argument can remove this assumption.

Theorem 1 (cf. Geck [Ge III]) Let $G$ be a simple algebraic group of type $\left(E_{7}\right)$, defined over $\mathbb{F}_{q}$, with Frobenius map $F$. Let $\rho$ be a (complex) cuspidal unipotent representation of $G^{F}$ with character $\chi_{\rho}$. Then the value field $\mathbb{Q}\left(\chi_{\rho}\right)$ of $\chi_{\rho}$ is $\mathbb{Q}\left(\sqrt{-q^{7}}\right)$. (i) If $p=2$, or $q$ is an odd power of $p$, or $q$ is an even power of $p$ such that $p \equiv 3(\bmod 4)$, then $m_{\mathbb{Q}}(\rho)=1$. (ii) Assume that $q$ is an even power of $p$ such that $p \equiv 1(\bmod 4)$, Then we have $m_{\mathbb{Q}_{\infty}}(\rho)=$ $m_{\mathbb{Q}_{\ell}}(\rho)=1$ for any prime number $\ell \neq p$ and $m_{\mathbb{Q}_{p}}(\rho)=2$. Thus $m_{\mathbb{Q}}(\rho)=2$.

By Propositions 5.5, 5.6 of [Ge I], we see that a unipotent representations of $E_{8}(q)$ with character $E_{7}[\xi], 1, E_{7}[-\xi], 1, E_{7}[\xi], \varepsilon$ or $E_{7}[-\xi], \varepsilon$ has the same rationality.

Remark Let $h\left(X_{f, 0}\right)$ be the motive over $\mathbb{F}_{q}$ corresponding to $X_{f, 0}^{\cdot}$ (see Milne [Mi II]), and let $Z$ be the simple submotive of $h\left(X_{f, 0}^{\cdot}\right)$ such that $\left[\pi_{Z}\right]=\left[\sqrt{-q^{7}}\right]$ (cf. [Mi II, Proposition 2.6]). Then we see from Theorem 2.16 of $[\mathrm{Mi} \mathrm{II}]$ that the distribution of the Hasse invariants of the division algebra $\operatorname{End}(Z)$ coincides with the results of Theorem 1.

3.

Let $G$ be a simple algebraic group, defined over $\mathbb{F}_{q}$, with Frobenius map $F$. Let $\delta$ be the minimal natural number such that $F^{\delta}$ acts trivially 
on $W_{G}$.

Let $\underline{s}=\left(s_{1}, \ldots, s_{n}\right)$ be a sequence of simple reflections in $W_{G}$, and let

$$
\begin{aligned}
X_{\underline{s}}=X\left(s_{1}, \ldots, s_{n}\right)=\{ & \left(B_{0}, B_{1}, \ldots, B_{n}\right) \in X_{G}^{n+1} \mid\left(B_{i-1}, B_{i}\right) \in s_{i} \\
& \text { for } \left.1 \leq i \leq n \text { and } F\left(B_{0}\right)=B_{n}\right\} .
\end{aligned}
$$

Then $X_{\underline{s}}$ is a locally closed subvariety of $X_{G}^{n+1}$ on which $G^{F}$ acts diagonally. We can prove that, for each $i$, each irreducible component of the $G^{F}$-module $H_{c}^{i}\left(X_{\underline{s}}\right)$ is unipotent (We use [Lu II, p. 25-6] and [DL, Theorem 6.2]).

Let $\rho$ be a unipotent representation of $G^{F}$. Then we have $\left(R^{1}(w), \rho\right)_{G^{F}} \neq$ 0 for some $w \in W_{G}$. We note that $R^{1}(w)=\sum_{i}(-1)^{i} H_{c}^{i}(X(w))$. Let $w=$ $s_{1} \cdots s_{n}$ be a reduced expression for $w(n=\ell(w))$. Then $X(w)$ is isomorphic to $X_{\underline{s}}$ with $\underline{s}=\left(s_{1}, \ldots, s_{n}\right)$. Therefore there is an integer $i$ such that $\left(H_{s}^{i}\left(\bar{X}_{\underline{s}}\right), \rho\right)_{G^{F}} \neq 0$.

Let $\underline{s}=\left(s_{1}, \ldots, s_{n}\right)$ be a minimal sequence such that $\left(H_{c}^{i}\left(X_{\underline{s}}\right), \rho\right)_{G^{F}} \neq 0$ for some $i$. Then we see that $\ell\left(s_{1} \cdots s_{n}\right)=n$ and $X_{\underline{s}} \stackrel{\sim}{\rightarrow} X(w)$ with $w=$ $s_{1} \cdots s_{n}$ (cf. [Lu II, pp. 25-6]). In the following, we fix one of such $\underline{s}$.

We have $\left(H_{c}^{i}\left(X_{\underline{s}}\right), \rho\right)_{G^{F}}=0$ for $i \neq n$ (Haastert [Ha, Korollar 4.4 (1)]). Therefore $w$ is an element of $W_{G}$ with minimal length such that $\left(R^{1}(w), \rho\right)_{G^{F}} \neq 0$. If $\rho$ is cuspidal, then $\left(R^{1}(w), \rho\right)_{G^{F}}=(-1)^{r}$, where $r$ is the $\mathbb{F}_{q}$-rank of $G$ (Lusztig [Lu V]).

Let

$$
\begin{gathered}
\bar{X}_{\underline{s}}=\bar{X}\left(s_{1}, \ldots, s_{n}\right)=\left\{\left(B_{0}, B_{1}, \ldots, B_{n}\right) \in X_{G}^{n+1} \mid\left(B_{i-1}, B_{i}\right) \in s_{i} \cup e\right. \\
\text { for } \left.1 \leq i \leq n \text { and } F\left(B_{0}\right)=B_{n}\right\} .
\end{gathered}
$$

Then $\bar{X}_{\underline{s}}$ is a smooth closed subvariety of $X_{G}^{n+1}$ ([DL, 9.10]) and $X_{\underline{s}}$ is an open dense subvariety of $\bar{X}_{\underline{s}}$. By the minimality of $\underline{s}$, we see that the inclusion $X_{\underline{s}} \hookrightarrow \bar{X}_{\underline{s}}$ induces an isomorphism as $G^{F}$-modules from $\rho$-isotropic part $H_{c}^{n}\left(X_{\underline{s}}\right)_{\rho}$ of $H_{c}^{n}\left(X_{\underline{s}}\right)$ onto the $\rho$-isotropic part $H^{n}\left(\bar{X}_{\underline{s}}\right)_{\rho}$ of $H^{n}\left(\bar{X}_{\underline{s}}\right)$ ([Lu II, p. 26]).

Let $X^{\cdot}=\bar{X}_{\underline{s}}$. Let $m$ be any multiple of $\delta$, and let $N_{\underline{s}}^{m}\left(g_{0}\right)=\left|X^{\cdot} g_{0} F^{m}\right|$ $\left(g_{0} \in G^{F}\right)$. Then we have (Digne and Michel [DM, pp. 60-61]):

$$
N_{\underline{s}}^{m}\left(g_{0}\right)=\sum_{i=0}^{2 n}(-1)^{i} \operatorname{Tr}\left(\left(g_{0} F^{m}\right)^{*}, H^{i}\left(X^{\cdot}\right)\right)
$$




$$
\begin{aligned}
& =\sum_{i=0}^{2 n}(-1)^{i} \operatorname{Tr}\left(\left(F^{\delta}\right)^{* m / \delta}\left(g_{0}\right)^{*}, H^{i}\left(X^{\cdot}\right)\right) \\
& =\sum_{i=0}^{2 n}(-1)^{i} q^{m i / 2} \sum_{\rho^{\prime} \in U}\left(H^{i}\left(X^{*}\right), \rho^{\prime}\right)_{G^{F}} \omega_{\rho^{\prime}}^{m / \delta} \chi_{\rho^{\prime}}\left(g_{0}\right) .
\end{aligned}
$$

(Note that one can prove that any irreducible component of $H^{i}\left(X^{\cdot}\right)$ is unipotent (cf. [Lu II, p. 26].).) Here $U$ is the set of isomorphism classes of the unipotent representations of $G^{F}$ and, for $\rho^{\prime} \in U, \omega_{\rho^{\prime}}$ is a root if unity such that $\omega_{\rho^{\prime}} q^{i \delta / 2}$ is the eigenvalue of $\left(F^{\delta}\right)^{*}$ on $H^{i}\left(X^{\prime}\right)$ associated with $\rho^{\prime}$ ([Lu II $]$ ).

Suppose that $\rho$ is cuspidal. Then $X^{\cdot}$ is irreducible (Lusztig [Lu II, pp. 26-27]). Let $W\left(\mathbb{F}_{q^{\delta}}\right)$ be the ring of Witt vectors over $\mathbb{F}_{q^{\delta}}$, let $K$ be its quotient field and let $\bar{K}$ be an algebraic closure of $K$. Let $X_{0}^{\cdot}$ be the $\mathbb{F}_{q^{\delta}}$-rational structure on $X^{\cdot}$ determined by the Frobenius $F^{\delta}: X^{\cdot} \rightarrow X^{\cdot}$. Let $F_{0}: X_{0} \rightarrow X_{0}^{\cdot}$ be the Frobenius endomorphism of $X_{0}^{\cdot}\left(F_{0}=\left(F_{\text {abs }}\right)^{a^{\prime} \delta}\right.$, $\left.q=p^{a^{\prime}}\right)$. Then, by Theorem 2 of $[\mathrm{KM}]$, we have

$$
\operatorname{Tr}\left(\left(g_{0} F_{0}^{m}\right)^{*}, H_{\text {crys }}^{i}\left(X_{0}^{*}\right)\right)=\operatorname{Tr}\left(\left(g_{0} F^{m}\right)^{*}, H^{i}\left(X^{\cdot}\right)\right) \quad(i \geqq 0) .
$$

Let $\alpha$ be an eigenvalue of $\left(F_{0}\right)^{*} \otimes 1$ on $H_{\text {crys }}^{i}\left(X_{0}^{\cdot}\right) \otimes_{K} \bar{K}$ and let $H_{\text {crys }}^{i}\left(X_{0}^{\cdot}\right)_{\alpha}$ be the generalized $\alpha$-eigensubspace of $H_{\text {crys }}^{i}\left(X_{0}^{*}\right) \otimes_{K} \bar{K} . \quad H_{\text {crys }}^{i}\left(X_{0}^{*}\right)_{\alpha}$ is a $\bar{K}\left[G^{F}\right]$-submodule of $H_{\text {crys }}^{i}\left(X_{0}^{\dot{*}}\right) \otimes_{K} \bar{K}$. In views of (2), (3), together with Grothendieck's trace formula for the étale cohomology, we see, by using the linearly independence of the irreducible characters of $G^{F}$ and the linearly independence of the functions $m / \delta \rightarrow \omega_{\rho^{\prime}}^{m / \delta}$, that if $\rho^{\prime}$ is contained in $H_{\text {crys }}^{i}\left(X_{0}^{*}\right)_{\alpha}$, then $\alpha$ is of the form $\omega_{\rho^{\prime}} q^{i \delta / 2}$.

Assume that $G$ is of type $\left(E_{8}\right)$ and that $\rho$ is a cuspidal unipotent representation of $G^{F}$ such that $\chi_{\rho}=E_{8}[i]$ or $E_{8}[-i]$. Then $\mathbb{Q}\left(\chi_{\rho}\right)=\mathbb{Q}(i)([$ Ge I, $\S 5])$ and $n=\ell(w)=10([\mathrm{Lu} \mathrm{V}])$. Therefore, by Hasse's sum formula, we get $m_{\mathbb{Q}_{p}}(\rho)=1$ if $p=2$ or $p \equiv 3(\bmod 4)$.

Assume that $p \equiv 1(\bmod 4)$. Then we have $\mathbb{Q}_{p}\left(\chi_{\rho}\right)=\mathbb{Q}_{p}(i)=\mathbb{Q}_{p}$, and we see that, by taking $M=H_{\text {crys }}^{10}\left(X_{f, 0}^{\cdot}\right)_{\rho},(M, \phi)$ is an isocrystal over $K$. Thus, by considering the representation

$$
R: K\left[G^{F}\right] \longrightarrow \operatorname{End}_{K}(M),
$$

the argument goes as $\S 2$ (note that we see that $(M, \phi)$ is a semisimple isocrystal). Thus we have $m_{\mathbb{Q}_{p}}(\rho)=1$, hence $m_{\mathbb{Q}}(\rho)=1$. 
In the following theorem, the case where $p=5$ was discussed in [Ge II] and $[\mathrm{He}]$ in an individual way, as explained in Introduction. Out method gives a uniform and conceptual proof in the case $p \equiv 1(\bmod 4)$.

Theorem 2 (cf. Geck [Ge I, II] and Hezard [He]) The cuspidal unipotent characters $E_{8}[ \pm i]$ of $E_{8}(q)$ have the Schur index 1 over $\mathbb{Q}$.

The same argument can be applied to any unipotent cuspidal representation $\rho$ with $\mathbb{Q}_{p}\left(\chi_{\rho}\right)=\mathbb{Q}_{p}$ for any $G$. Therefore it remains the case where $G$ is of type $\left(E_{8}\right)$ and $\rho$ is such that $\chi_{\rho}=E_{8}\left[\zeta^{j}\right](1 \leq j \leq 4), p \equiv 4(\bmod 5)$. But, in this case, we can argue as follows.

Let $\chi=\chi_{\rho}=E_{8}\left[\zeta^{j}\right]$, and let $\chi^{\prime}$ be the algebraically conjugate character of $\chi$ over $\mathbb{Q}_{p}$, i.e. $\chi^{\prime}=E_{8}\left[\zeta^{4 j}\right]$. Since the character of the $K\left[G^{F}\right]$ module $H_{\text {crys }}^{n}\left(X_{0}^{*}\right)$ takes values in $\mathbb{Z}$. we must have $\left(H_{\text {crys }}^{n}\left(X_{0}^{\cdot}\right), \rho^{\prime}\right)_{G^{F}}=$ $\left(H_{\text {crys }}^{n}\left(X_{0}^{*}\right), \rho\right)_{G^{F}}=1$, where $\rho^{\prime}$ is a representation of $G^{F}$ with character $\chi^{\prime}$. Therefore, by the property of the Schur index, we have $m_{K}(\rho)=$ $m_{K}\left(\rho^{\prime}\right)=1$, so that, by a threorem of Schur, we see that $\rho \oplus \rho^{\prime}$ is a representation of $G^{F}$ which is realizable in $K$. Hence there is a unique submodule $M$ of $H_{\text {crys }}^{n}\left(X_{0}^{*}\right)$ with character $\chi+\chi^{\prime}$. We must have $\phi(M)=M$, since $\phi(M)$ is a $G^{F}$-submodule of $H_{\text {crys }}^{n}\left(X_{0}^{*}\right)$ with character $\sigma\left(\chi+\chi^{\prime}\right)=\chi+\chi^{\prime}$. Thus $(M, \phi)$ is an isocrystal over $K$.

Let us consider the representation

$$
R: K\left[G^{F}\right] \longrightarrow \operatorname{End}_{K}(M) .
$$

Let $A\left(\chi, \mathbb{Q}_{p}\right)$ be the simple component of $\mathbb{Q}_{p}\left[G^{F}\right]\left(\subset K\left[G^{F}\right]\right)$ associated with $\chi$. Then we see that $R\left(\mathbb{Q}_{p}\left[G^{F}\right]\right)=R\left(A\left(\chi, \mathbb{Q}_{p}\right)\right)$ (cf. T. Yamada [Ya, Proposition 1.1 , pp. $4-5])$. Then since $A\left(\chi, \mathbb{Q}_{p}\right)$ is a central simple algebra over $\mathbb{Q}_{p}(\chi)=\mathbb{Q}_{p}(\zeta)$ and $R$ is a ring homomorphism, we see that $B=$ $R\left(\mathbb{Q}_{p}\left[G^{F}\right]\right)$ is a simple algebra, isomorphic to $A\left(\chi, \mathbb{Q}_{p}\right)$. By Lemma 2 for $X_{0}$, we must have $B \subset \operatorname{End}(M, \phi)$.

We have $M \otimes_{K} \bar{K}=M_{\rho} \oplus M_{\rho^{\prime}}$, where $M_{\rho}$ (resp. $M_{\rho^{\prime}}$ ) is the $\rho$-isotropic part (resp. $\rho^{\prime}$-isotropic part) of $M \otimes_{K} \bar{K}$. Let $\pi_{M}=\phi^{a^{\prime}}=\left(F_{0}\right)^{*} \otimes 1\left(q=p^{a^{\prime}}\right)$ on $M$. The eigenvalues of $\left(F_{0}\right)^{*} \otimes 1$ on $M_{\rho} \subset\left(M \otimes_{K} \bar{K}\right)_{\zeta^{j} q^{n / 2}}$ (resp. $M_{\rho^{\prime}} \subset$ $\left.\left(M \otimes_{K} \bar{K}\right)_{\zeta^{4 j} q^{n / 2}}\right)$ are of the form $\zeta^{j} q^{n / 2}$ (resp. $\left.\zeta^{4 j} q^{n / 2}\right)$. Since the actions of $\left(F_{0}\right)^{*} \otimes 1$ and $G^{F}$ commute, by Schur's lemma, we must have $\left(F_{0}\right)^{*} \otimes 1=$ $\zeta^{j} q^{n / 2}\left(\right.$ resp. $\left.=\zeta^{4 j} q^{n / 2}\right)$ on $M_{\rho}$ (resp. $\left.M_{\rho^{\prime}}\right)$. Therefore the endomorphism $\pi_{M}$ of $M$ is semisimple, hence $(M, \phi)$ is a semisimple isocrystal over $K$ (see Milne [Mi II, Proposition 2.10, p. 417]). Therefore $\operatorname{End}(M, \phi)$ is a $\mathbb{Q}_{p}$-form 
on the centralizer $C=Z_{E}\left(\pi_{M}\right)$ of $\pi_{M}$ in $E=\operatorname{End}_{K}(M)$ ([Ko, p. 410]). We have $C \otimes_{K} \bar{K} \subset Z_{E \otimes_{K} \bar{K}}\left(\pi_{M}\right) \cong M_{d}(\bar{K}) \oplus M_{d}(\bar{K})$, where $d=\chi(1)=$ $\chi^{\prime}(1)$, and it is well known that $B \otimes_{K} \bar{K} \simeq A\left(\chi, \mathbb{Q}_{p}\right) \otimes_{K} \bar{K}=M_{d}(\bar{K}) \oplus$ $M_{d}(\bar{K})$. Therefore we must have $B=\operatorname{End}(M, \phi)$. Therefore, as $B$ is simple and $(M, \phi)$ is semisimple, there is a simple subisocrystal $(X, \phi)$ of $(M, \phi)$ such that $B=\operatorname{End}(M, \phi) \simeq M_{t}(D)$ with $D=\operatorname{End}(X, \phi)$ for some positive integer $t$. By Lemma 11.3 of $[\mathrm{Ko}]$, we see that the Hasse invariant of $D$ can be given by $-\left(\operatorname{ord}_{p}\left(\pi_{X}\right) / \operatorname{ord}_{p}(q)\right)\left[\mathbb{Q}_{p}\left(\pi_{X}\right): \mathbb{Q}_{p}\right]$, where $\operatorname{ord}_{p}$ is the valuation of $\mathbb{Q}_{p}$ and its extension to the field $\mathbb{Q}_{p}\left[\pi_{X}\right]$ and $\pi_{X}=\phi^{a^{\prime}}$ on $X$. But $\mathbb{Q}_{p}\left[\pi_{X}\right] \cong \mathbb{Q}_{p}(\chi) \cong \mathbb{Q}_{p}(\zeta)$ and $\operatorname{ord}_{p}\left(\pi_{X}\right)=a^{\prime} n / 2, \operatorname{ord}_{p}(q)=a^{\prime}$, hence

$$
\operatorname{inv}\left(A\left(\chi, \mathbb{Q}_{p}\right)\right) \equiv-\frac{n}{2}\left[\mathbb{Q}_{p}(\chi): \mathbb{Q}_{p}\right] \equiv-\frac{r}{2}\left[\mathbb{Q}_{p}(\chi): \mathbb{Q}_{p}\right] \equiv 0 \quad(\bmod 1)
$$

(note that $\left.(-1)^{n}=(-1)^{r}\right)$. Thus $m_{\mathbb{Q}_{p}}(\rho)=1$ and $m_{\mathbb{Q}}(\rho)=1$.

Remark The last argument works in general case ( $G$ is simple, $\rho$ is cuspidal, and $q, p$ arbitrary). Therefore we can prove Theorem $\mathrm{A}$ in the introduction.

"Theorem B" follows from this proof of Theorem A and Theorem 2.16 of Milne [Mi II].

Acknowledgement I wish to thank the referee for his (her) kind comments of the original versions of the paper. Finally, I wish to dedicate this paper to my daughters Chieko and Fumiko.

\section{References}

[Ber] Berthelot F., Cohomologie cristalline des schémas de charactéristique $p>0$. Lecture Notes in Mathematics, vol. 407, Springer-Verlag, 1974.

[BO] Berthelot P. and Ogus A., Note on crystalline cohomology. Mathematical Notes, Princeton University Press, 1978.

[Ca] Carter C.W., Finite groups of Lie type - Conjugacy classes and complex characters. Interscience Publication, John Wiley and Sons, 1985.

[CE] Cartan H. and Eilenberg S., Homological algebra. Princeton Mathematical Series No. 19, Princeton University Press, 1956.

[CR] Curtis C.W. and Reiner I., Representation theory of finite groups and assocative algebras. Interscience Publication, John-Wiley and Sons, 1962.

[De] Deligne P., La conjecture de Weil I. Inst. Hautes Etudes Sci. Publ. Math. 43 (1974), 273-307.

[DL] Deligne P. and Lusztig G., Representation of reductive groups over finite fields. Ann. of Math. 103 (1976), 103-161. 
[DM] Deligne P. and Michel J., Fonctions L des variétés de Deligne-Lusztig et descent de Shintani. Memorie de la Societé Mathématique de France, no. 20, 1985.

[Ge I] Geck M., Character values, Schur indices and character sheaves. Representation Theory 7 (2003), 19-55.

[Ge II] Geck M., On the Schur indices of cuspidal unipotent characters. arXiv: In Finite Groups 2003, 87-104, Walten de Gruyter Grusblt \& Co. KG, Berlin, 2004.

[Ge III] Geck M., The Schur indices of the cuspidal unipotent characters of the finite Chevalley groups $E_{7}(q)$. Osaka J. Math. 42 (2005), 201-215.

[Ha] Haastert B., Die Quasiaffintät der Deligne-Lusztig Varietäten. J. Algebra 102 (1986), 196-193.

[He] Hezard D., Ph. D. thesis, Universite Lyon 1.

[Ill] Illusie L., Crystalline cohomology. Proceedings of Symposia in Pure Mathematics 55 (1994), Part I, 43-70.

[KM] Katz N. and Messing W., Some consequences of the Riemann hypothesis for varieties over finite fields. Invent. Math. 23 (1974), 73-77.

[Kl] Kleimann S.L., The standard conjectures. Proceedings of Symposia in Pure Mathematics 55 (1994), Part I, 3-20.

[Ko] Kottwitz R.E., Points on some Shimura varieties over finite fields. J. American Mathematical Society 5 (1992), 373-444.

[Lu I] Lusztig G., Coxeter orbits and eigenspace of Frobenius. Invernt. Math. 38 (1976), 101-159.

[Lu II] Lusztig G., Representations of finite Chevalley groups. Conference Board of mathematical science regional conference series in mathematics, number 19, AMS, 1976.

[Lu III] Lusztig G., Characters of reductive groups over a finite field. Ann. of Math. Studies 107, Princeton University Press, 1984.

[Lu IV] Lusztig G., A unipotent support for irreducible representations. Advances in Math. 94 (1992), 139-179.

[Lu V] Lusztig G., Rationality properties of unipotent representations. J. Algebra 258 (2002), 1-22.

[Mi I] Milne J.S., Étale cohomology. Princeton, 1980.

[Mi II] Milne J.S., Motives over finite fields. Proceedings of Symposia in Pure Mathematics 55 (1994), Part I, 401-459.

[Sri] Srinivasan B., Representations of finite Chevalley groups. Lecture Notes in Mathematics, vol. 764, Springer-Verlag, 1979.

[Ya] Yamada T., The Schur subgroup of the Brauer group. Lecture Notes in Mathematics, vol. 397, Springer-Verlag, 1974.

Mathematical Department Hokkaido University of Education Iwamizawa Campus, Midorigaoka Iwamizawa 068-8642, Hokkaido, Japan 